\title{
Le syndrome inflammatoire multisystémique de l'enfant ayant un lien temporel avec la COVID-19 : multiples visages, multiples appellations
}

\author{
Herman Tam MBBS MSc, Tala El Tal MD, Ellen Go MD, Rae SM Yeung MD PhD
}

Citation : CMAJ 2020 September 21;192:E1093-6. doi : 10.1503/cmaj.201600-f; diffusion hâtive le 9 septembre 2020

Entrevue avec l'auteur (en anglais) en baladodiffusion : https://www.cmaj.ca/lookup/doi/10.1503/cmaj.201600/tab-related-content

Voir la version anglaise de l'article ici : www.cmaj.ca/lookup/doi/10.1503/cmaj.201600

$\mathbf{U}$

n garçon de 10 ans jusqu'ici en santé arrive à l'urgence.

Il a une fièvre qui persiste depuis 6 jours; se plaint de douleurs à l'abdomen et a des vomissements et la diarrhée depuis 4 jours; présente une conjonctivite bilatérale non purulente; et a les lèvres rouges et fendillées. Quatre semaines auparavant, le patient a souffert de céphalées pendant 3 jours, sans symptômes respiratoires, et sa famille et lui ont testé positif au dépistage du coronavirus du syndrome respiratoire aigu sévère 2 (SRAS-CoV-2) effectué sur un échantillon prélevé par écouvillonnage du nasopharynx. À son arrivée, le garçon a une pression artérielle de $74 / 35 \mathrm{~mm} \mathrm{Hg}$ et une fréquence cardiaque de 130 battements par minute, malgré une réanimation liquidienne correspondant à $60 \mathrm{~mL} / \mathrm{kg}$. Son corps est froid, et sa circulation sanguine, mauvaise. Nous lui administrons un agent inotrope et un traitement antibiotique empirique, puis le transférons aux soins intensifs.

Les résultats des analyses de laboratoire effectuées à l'admission (encadré 1) sont cliniquement significatives pour les anomalies et affections suivantes : hyperleucocytose, neutrophilie, lymphopénie, thrombopénie, élévation du taux de protéines $C$ réactives (CRP), hyperferritinémie, hyponatrémie, hypoalbuminémie, hypertriglycéridémie, insuffisance rénale aiguë, transaminite, coagulopathie et nette élévation des taux de troponine et de propeptide $\mathrm{N}$-terminal du peptide natriurétique de type B (NT-proBNP). L'électrocardiogramme révèle une anomalie de conduction, et l'échocardiogramme montre des signes de myocardite, sans changement ou anévrisme des artères coronaires. Le test de dépistage du SRAS-CoV-2 par réaction en chaîne par polymérase (PCR) effectué sur un échantillon prélevé par écouvillonnage du nasopharynx est négatif, mais le test sérologique est positif. Nous posons un diagnostic de syndrome inflammatoire multisystémique de l'enfant caractérisé par des particularités associées à la maladie de Kawasaki, au choc cardiogénique, à la myocardite, à la dysfonction hépatique, à l'insuffisance rénale aiguë et à un syndrome d'activation macrophagique progressif.

\section{POINTS CLÉS}

- Le syndrome inflammatoire multisystémique de l'enfant est un trouble hyperinflammatoire nouveau et rare ayant un lien temporel avec la maladie à coronavirus 2019 (COVID-19); ses tableaux cliniques, très variables sur le plan de la gravité, sont regroupés en 3 grands types : « choc », «maladie de Kawasaki », et « fièvre et inflammation ».

- Ses principaux signes cliniques sont une fièvre prolongée; les caractéristiques typiques de la maladie de Kawasaki; un dysfonctionnement cardiaque; et des symptômes gastrointestinaux, neurologiques et/ou rénaux.

- En laboratoire, l'hyperinflammation associée se manifeste entre autres par une élévation notable du taux de protéines $C$ réactives, une lymphopénie, une neutrophilie, une thrombopénie, une hyponatrémie et une hypoalbuminémie.

- Les immunomodulateurs utilisés pour la maladie de Kawasaki, comme les immunoglobulines à administration intraveineuse et les glucocorticoïdes, sont des options de traitement envisageables.

Dès l'admission du patient, nous lui administrons un traitement combinant l'injection intraveineuse de bolus de méthylprednisolone équivalents à $30 \mathrm{mg} / \mathrm{kg} / \mathrm{j}$ pour 4 jours, suivis d'un sevrage très graduel, et l'injection intraveineuse d'une dose d'immunoglobulines $(2 \mathrm{~g} / \mathrm{kg})$. Nous cessons l'administration de l'agent inotrope le $4^{e}$ jour. En raison de la fièvre persistante et de la cytopénie du garçon, nous ajoutons, le $7^{\mathrm{e}}$ jour, l'administration de $100 \mathrm{mg} / \mathrm{j}$ (correspondant à environ $2,75 \mathrm{mg} / \mathrm{kg} / \mathrm{j}$ ) d'anakinra, une forme recombinante de l'antagoniste du récepteur de l'interleukine-1 (IL-1). Sa fièvre disparaît le jour même. Une fois sa thrombopénie résorbée, le $9^{\mathrm{e}}$ jour, nous commençons à lui administrer de faibles doses d'acide acétylsalicylique (dose d'anticoagulants : 3 à $5 \mathrm{mg} / \mathrm{kg} / \mathrm{j}$ ). Sa fonction rénale et son taux de troponine reviennent à la normale respectivement le $3^{e}$ et le $8^{\mathrm{e}}$ jour. Le $10^{\mathrm{e}}$ jour, ses symptômes cliniques et ses résultats d'analyses biochimiques s'améliorent encore. Nous lui donnons 
son congé, et lui prescrivons de l'anakinra et un protocole de sevrage de la prednisone. Au total, le patient passe 10 jours à l'hôpital, dont 5 aux soins intensifs.

À la rencontre de suivi, on constate que le garçon se porte toujours bien et que ses marqueurs inflammatoires s'améliorent. Il présente cependant une thrombocytose - son taux de plaquettes

\section{Encadré 1 : Analyses de laboratoire et autres tests effectués à l'admission}

\begin{tabular}{|c|c|c|}
\hline Analyse ou test & Résultat & $\begin{array}{l}\text { Valeurs de } \\
\text { référence }\end{array}$ \\
\hline Globules blancs, $\times 10^{9} / \mathrm{L}$ & 13,41 & $4,31-11,00$ \\
\hline $\begin{array}{l}\text { Neutrophiles (nombre } \\
\text { absolu), } \times 10^{9} / \mathrm{L}\end{array}$ & 8,70 & $1,63-7,55$ \\
\hline $\begin{array}{l}\text { Lymphocytes (nombre } \\
\text { absolu), } \times 10^{9} / \mathrm{L}\end{array}$ & 0,36 & $>3$ \\
\hline Hémoglobine, g/dL & 101 & $107-134$ \\
\hline Plaquettes, $\times 10^{9} / \mathrm{L}$ & 30 & $206-369$ \\
\hline Protéines $C$ réactives, $\mathrm{mg} / \mathrm{L}$ & 268,9 & $0,1-1,0$ \\
\hline VSE, mm/h & 9 & $2-34$ \\
\hline Ferritine, $\mathrm{ng} / \mathrm{mL}$ & 1061,5 & $13,7-78,8$ \\
\hline Sodium, mmol/L & 132 & $135-143$ \\
\hline Albumine, $\mathrm{g} / \mathrm{dL}$ & 22 & $37-50$ \\
\hline Alanine aminotransférase, $\mathrm{U} / \mathrm{L}$ & 81 & $<24$ \\
\hline $\begin{array}{l}\text { Aspartate aminotransférase, } \\
\mathrm{U} / \mathrm{L}\end{array}$ & 84 & $<43$ \\
\hline Créatinine, $\mu \mathrm{mol} / \mathrm{L}$ & 182 & $25-50$ \\
\hline D-dimères, $\mu \mathrm{g} / \mathrm{mL}$ & 3,35 & $<0,50$ \\
\hline Fibrinogène, $\mathrm{g} / \mathrm{L}$ & 6,6 & $1,9-4,3$ \\
\hline $\begin{array}{l}\text { Rapport international } \\
\text { normalisé }\end{array}$ & 1,4 & $0,8-1,2$ \\
\hline Triglycérides, mmol/L & 3,35 & $<1,02$ \\
\hline Troponine I, ng/L & 199,7 & $<30,9$ \\
\hline NT-proBNP, pmol/L & 2960 & $<150$ \\
\hline $\begin{array}{l}\text { SRAS-CoV-2, dépistage par } \\
\text { PCR sur un échantillon } \\
\text { prélevé par écouvillonnage } \\
\text { du nasopharynx }\end{array}$ & Négatif & S.O. \\
\hline $\begin{array}{l}\text { IgG dirigées contre le } \\
\text { SRAS-CoV-2 }\end{array}$ & Positif & S.O. \\
\hline Électrocardiogramme & $\begin{array}{l}\text { Tachycardie sinusale, } \\
\text { retard de conduction } \\
\text { dans le VD }\end{array}$ & S.O. \\
\hline Échocardiogramme & $\begin{array}{l}\text { Artères coronaires } \\
\text { normales; diminution } \\
\text { modérée de la fonction } \\
\text { du VD; zones claires au } \\
\text { myocarde et au muscle } \\
\text { papillaire, du côté du } \\
\text { VG; léger épanchement } \\
\text { péricardique }\end{array}$ & S.O. \\
\hline \multicolumn{3}{|c|}{$\begin{array}{l}\text { Remarque : IgG = immunoglobulines G, NT-proBNP = propeptide N-terminal du peptide } \\
\text { natriurétique de type B, PCR = réaction en chaîne par polymérase, } S .0 \text {. = sans objet, } \\
\text { SRAS-CoV-2 = coronavirus du syndrome respiratoire aigu sévère } 2, V D=\text { ventricule } \\
\text { droit, VG = ventricule gauche, VSE = vitesse de sédimentation érythrocytaire. }\end{array}$} \\
\hline
\end{tabular}

a atteint $545 \times 10^{9} / \mathrm{L}-$ concomitante avec la desquamation sur les mains et des pieds, ce qui est caractéristique de la maladie de Kawasaki. L'électrocardiogramme et l'échocardiogramme montrent un fonctionnement normal, hormis un léger épanchement péricardique. Le sevrage des stéroïdes s'est terminé après 3 semaines de traitement, mais le patient prend toujours de l'anakinra au moment où nous écrivons ces lignes.

\section{Discussion}

\section{Profil épidémiologique}

Les études épidémiologiques qui examinent la pandémie actuelle de maladie à coronavirus 2019 (COVID-19) partout dans le monde constatent que les enfants sont moins durement touchés que les adultes. Au Canada, les personnes de 19 ans et moins représentent $8,0 \%$ de l'ensemble des cas de COVID-19, mais 1,2\% des cas hospitalisés (en date du 4 août 2020, selon l'Agence de la santé publique du Canada; source accessible ici : https://sante -infobase.canada.ca/covid-19/resume-epidemiologique-cas-covid -19.html). La plupart des enfants dont le test est positif sont asymptomatiques ou ont des symptômes légers pendant la phase aiguë de l'infection. À Toronto, le nombre de cas de COVID-19 a commencé à grimper vers la fin mars et le début avril, et a été suivi 4 semaines plus tard d'une hausse du nombre de cas de syndrome inflammatoire multisystémique de l'enfant, qui venait alors d'être caractérisé.

\section{Définitions de cas}

Le 7 avril 2020, le premier cas de maladie de Kawasaki chez une personne ayant reçu un résultat positif au test de dépistage du SRAS-CoV-2 par PCR a été signalé. Il s'agissait d'une fillette de 6 mois présentant de la fièvre, des signes de la maladie de Kawasaki et des symptômes respiratoires très légers ${ }^{1}$. Le 25 avril 2020, le National Health Service du Royaume-Uni a envoyé aux médecins une alerte de santé publique pour les informer de l'émergence, chez des enfants, d'une affection inusitée caractérisée par une inflammation multisystémique provoquant des symptômes cardiaques et gastrointestinaux variés et ressemblant à la maladie de Kawasaki et au choc toxique staphylococcique. Depuis, le nombre de cas connaît une hausse alarmante, et différentes définitions et appellations ont été proposées pour désigner l'affection. On parle notamment de syndrome inflammatoire multisystémique de l'enfant ayant un lien temporel avec la COVID-192 , et de trouble inflammatoire multisystémique chez les enfants ${ }^{3,4}$. Certains éléments de ces définitions se recoupent : fièvre prolongée, dysfonctionnement de plusieurs organes, signes d'hyperinflammation observables en laboratoire, infection ou exposition ou non au SRAS-CoV-2, et absence d'autres causes pouvant expliquer le tableau clinique (encadré 2) ${ }^{5}$. Le 12 mai 2020, le Programme canadien de surveillance pédiatrique a publié une alerte de santé publique à ce sujet à l'intention des professionnels de la santé du pays ${ }^{6}$. On trouve maintenant des mentions de cas de ce nouveau syndrome hyperinflammatoire post-infectieux dans la littérature provenant des différents pays les plus touchés par la COVID-19, dont le Royaume-Uni, l'Italie, la France et les États-Unis ${ }^{7-10}$. 


\section{Lien épidémiologique avec le SRAS-CoV-2}

Bien que le syndrome inflammatoire multisystémique de l'enfant ait un lien épidémiologique avec la COVID-19, il est intéressant de voir que les définitions de cas n'exigent pas toutes des antécédents d'infection ou d'exposition au SRAS-CoV-2². Dans la plupart des cas décrits dans d'autres pays, les tests sérologiques étaient positifs, signe d'une infection au virus, mais seul un petit pourcentage d'enfants avaient un résultat positif au test par PCR effectué sur un échantillon prélevé par écouvillonnage du nasopharynx. Les autres critères liés à l'exposition sont la présence de symptômes pouvant correspondre à ceux d'une infection aiguë au SRAS-CoV-2 4 à 6 semaines auparavant, les soupçons ou la confirmation d'une exposition ou d'un contact, et le fait d'habiter dans une région comptant beaucoup de cas de COVID-19. Pour le moment, il n'existe pas de test sérologique clinique validé au Canada, car des normes nationales sont en train d'être établies. Il faudrait donc, dans le cadre de l'évaluation diagnostique avant traitement d'un cas soupçonné de syndrome inflammatoire multisystémique de l'enfant, prélever des échantillons de sérum à analyser lorsqu'un test sérologique sera sur le marché.

\section{Profil pathophysiologique}

On croit que l'inflammation et les lésions endothéliales causées par le SRAS-CoV-2 touchent principalement les vaisseaux sanguins ${ }^{11}$, ce qui correspond aux caractéristiques immunobiologiques de la maladie de Kawasaki. Même si les mécanismes pathophysiologiques exacts du syndrome inflammatoire multisystémique de l'enfant restent à déterminer, la présence

Encadré 2 : Comparaison des définitions de cas du syndrome inflammatoire multisystémique de l'enfant ${ }^{5}$

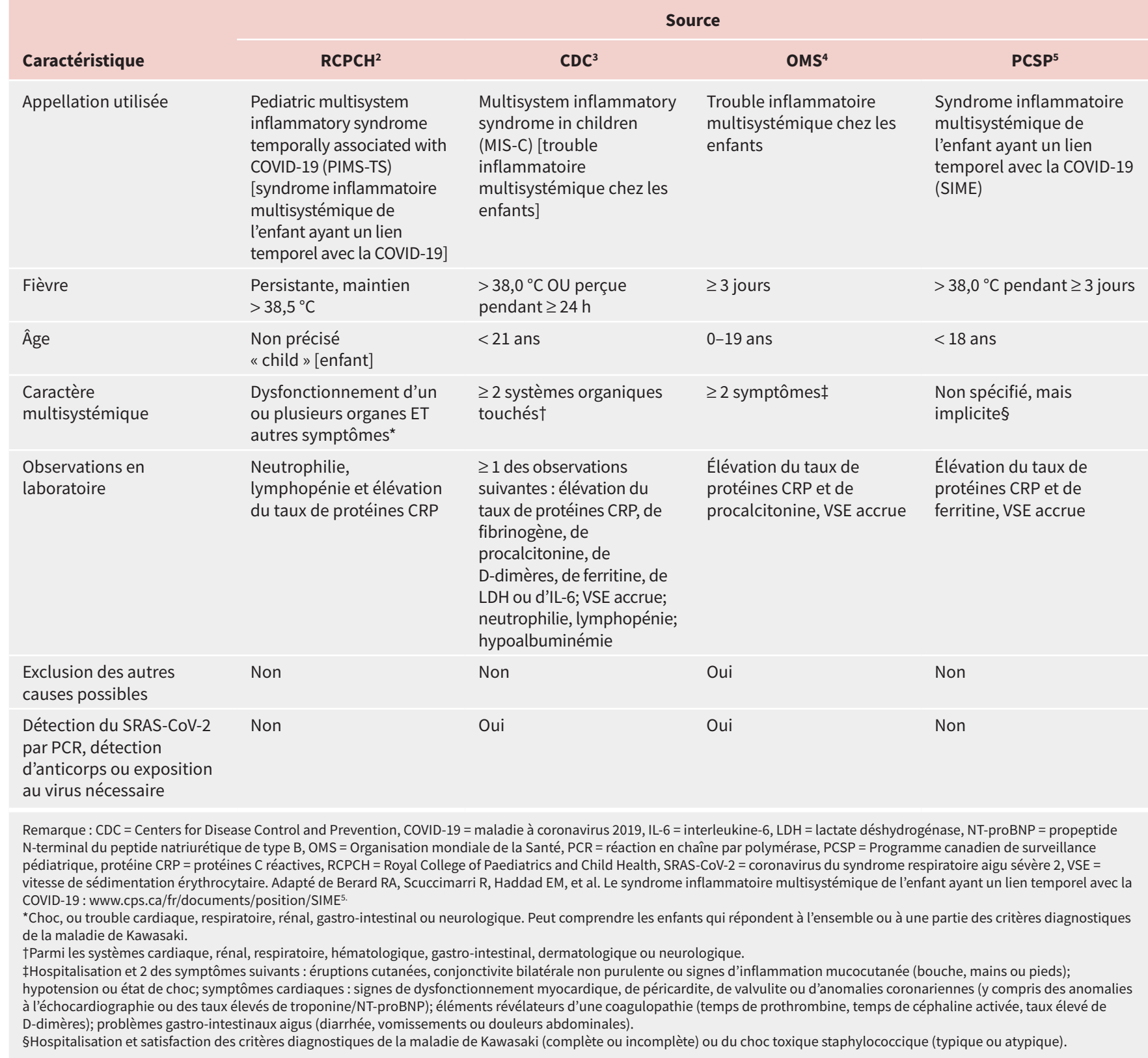


d'anticorps dirigés contre le SRAS-CoV-2, la réponse immunitaire spécifique médiée par les lymphocytes T et l'apparition des symptômes après le sommet de l'infection aiguë portent à croire que l'immunité acquise jouerait un rôle dans son développement, par exemple en favorisant la pénétration du virus dans les cellules ou en déclenchant une réponse pro-inflammatoire médiée par des anticorps ou des complexes immuns ${ }^{7,8}$. Cette possibilité a d'importantes implications pour la mise au point de vaccins et est activement étudiée par les chercheurs.

Dans les cas graves, le dérèglement de la réponse immunitaire peut engendrer une suractivité des macrophages, dérivés des monocytes, et faire augmenter la production de cytokines et de chimiokines, un peu comme on le voit avec le syndrome d'activation macrophagique ${ }^{5}$. Le syndrome d'activation macrophagique est la manifestation d'un choc cytokinique dû à l'activité et à la prolifération non régulées des lymphocytes $T$ et des macrophages. Il se caractérise par une fièvre persistante, une cytopénie, une hyperferritinémie, une dysfonction hépatique et une coagulopathie ${ }^{5}$. Les personnes atteintes ne se sentent pas bien : en raison d'une instabilité hémodynamique et d'atteintes à plusieurs organes, elles peuvent voir leur état se détériorer rapidement - un tableau clinique que l'on observe aussi dans le cas du syndrome inflammatoire multisystémique de l'enfant.

\section{Les multiples visages du syndrome inflammatoire multisystémique de l'enfant}

Comme la maladie de Kawasaki, le syndrome inflammatoire multisystémique de l'enfant regroupe différents syndromes aux tableaux cliniques extrêmement variés. Tous deux sont des syndromes hyperinflammatoires déclenchés par une infection qui peuvent avoir des complications allant d'un épisode de fièvre qui prend fin de lui-même, dans les cas légers, à un choc cardiogénique et un syndrome d'activation macrophagique, dans les cas graves. Pour le syndrome inflammatoire multisystémique de l'enfant, l'éventail de tableaux cliniques a été divisé en 3 grands types : le type « choc », le type « maladie de Kawasaki », et le type « fièvre avec inflammation ».

Le premier type, "choc », a beaucoup de signes cliniques communs avec le choc toxique staphylococcique et l'état de choc associé à la maladie de Kawasaki. Les enfants atteints sont très malades et en hypotension, et présentent un haut degré d'hyperinflammation (élévation notable du taux de protéines CRP, hyperferritinémie, neutrophilie, lymphopénie), une nette élévation des taux de marqueurs cardiaques (troponine et NT-proBNP), une hypoalbuminémie, une hyponatrémie, et des anomalies à l'électrocardiogramme ${ }^{7-10}$. Les méthodes de diagnostic par étapes dans les cas de syndrome inflammatoire multisystémique de l'enfant soupçonné devraient comprendre les analyses sanguines évoquées précédemment, qui peuvent aider à déterminer si le patient est très susceptible de faire un choc cardiogénique et guider la prise en charge. Le professionnel doit utiliser la réanimation liquidienne avec prudence, car une hyperhydratation est possible en cas de choc cardiogénique. II arrive aussi qu'il faille recourir aux soins intensifs, comme la ventilation mécanique et l'administration d'un agent inotrope.

Le deuxième type, "maladie de Kawasaki », est très similaire aux cas de maladie de Kawasaki non liés à une infection au SRAS-
CoV-2 : les enfants atteints font de la fièvre $\left(38,5^{\circ} \mathrm{C}\right)$ pendant 5 jours ou plus, et présentent les signes diagnostiques habituels de la maladie de Kawasaki (éruptions cutanées, injection conjonctivale sans exsudat, changements des muqueuses de la bouche [langue framboisée, lèvres rouges ou fendillées], changements périphériques [œdème ou rougeurs aux mains et aux pieds], lymphadénopathie cervicale, et inflammation vasculaire entraînant des dommages aux artères coronaires et la formation d'un anévrisme) ${ }^{12}$. Mais il y a aussi quelques différences. Le syndrome inflammatoire multisystémique touche des enfants plus vieux; est plus fréquent chez les enfants d'origine afrocaribéenne; provoque des symptômes gastro-intestinaux, neurologiques et rénaux majeurs; est associé à des affections cardiaques graves, comme la myocardite et le dysfonctionnement ventriculaire; et pose un risque accru de choc et de syndrome d'activation macrophagique $\mathrm{e}^{7-10}$.

Quant au dernier type, "fièvre et inflammation ", c'est le moins grave des 3 . Les enfants atteints font de la fièvre et montrent des signes biochimiques d'hyperinflammation, mais ne présentent aucune des caractéristiques cliniques de la maladie de Kawasaki ou du choc. À la rencontre de suivi, les patients vus à notre centre ont pour la plupart une thrombocytose marquée, une manifestation typique de la maladie de Kawasaki à la phase subaiguë ${ }^{12}$. Les 3 types peuvent occasionner des anévrismes des artères coronaires et des lésions de myocarde, même en l'absence de traits liés à la maladie de Kawasaki. Heureusement, quelques séries de cas ont fait état d'une disparation spontanée, sans traitement actif, des anévrismes des artères coronaires. Il est néanmoins essentiel de faire un suivi à long terme, afin de déterminer les interventions appropriées pour les patients qui n'ont pas d'anévrisme lors des premiers examens.

\section{Évaluation et prise en charge}

En juillet 2020, l'American College of Rheumatology (ACR $)^{13}$ et la Société canadienne de pédiatrie $(\mathrm{SCP})^{5}$ ont publié des lignes directrices cliniques sur l'évaluation diagnostique et la prise en charge des cas soupçonnés de syndrome inflammatoire multisystémique de l'enfant. Quand il pense être en présence de ce syndrome, le clinicien doit envisager la possibilité d'autres étiologies infectieuses et non infectieuses. Il doit aussi prendre en considération la prévalence locale de la COVID-19, puisque le syndrome a un lien temporel avec l'infection et l'exposition au SRASCoV-2. Étant donné le manque de données sur lesquelles baser les décisions, la plupart des lignes directrices jouent de prudence et ratissent large dans la détermination des patients à risque. Par exemple, la SCP propose de faire des tests de dépistage chez les enfants qui présentent, pendant plus de 3 jours, une fièvre inexpliquée dont les autres causes possibles ont été écartées ${ }^{5}$. Les documents seront revus fréquemment, au fur et à mesure que des données sortent, afin que les critères décisionnels permettent de détecter les cas tout en prévenant les analyses inutiles et en tenant compte de la disponibilité des ressources dans le milieu.

Pour le moment, aucune étude n'a fait de comparaison directe entre les divers traitements du syndrome inflammatoire multisystémique de l'enfant. La plupart du temps, la prise en charge est adaptée de celle de la maladie de Kawasaki, compte tenu des similitudes 
entre les 2 affections : utilisation d'immunomodulateurs, comme les immunoglobulines IV et les stéroïdes; thromboprophylaxie; et protocoles d'imagerie de surveillance cardiaque. Chez les patients qui ne tirent pas de bénéfices des immunoglobulines et des stéroïdes, la biothérapie, par exemple aux anti-IL-1, serait à envisager ${ }^{5,13}$. Enfin, l'évolution du traitement et la diminution de la dose d'immunosuppresseurs devraient suivre les résultats d'évaluations cliniques, d'analyses de laboratoire et de tests cardiaques répétés.

\section{Orientations futures}

Pour mieux comprendre ce nouveau syndrome et guider la prise de décisions cliniques, il nous faut appliquer une démarche scientifique rigoureuse dans la collecte et l'analyse des données, notamment s'entendre sur des définitions universelles. La SCP, en collaboration avec l'Agence de la santé publique du Canada, a élargi sa surveillance active de la COVID-19 pour inclure les cas de syndrome inflammatoire multisystémique de l'enfant et de maladie de Kawasaki, et ce, à l'échelle nationale ${ }^{6}$. Le Canada est dans une position enviable, car il peut apprendre de l'expérience des pays frappés par la COVID-19 avant lui. Nous sommes donc en mesure de faire connaître ce syndrome, et de créer et mettre en place des stratégies thérapeutiques pour les cas soupçonnés ${ }^{5}$.

\section{Références}

1. Jones VG, Mills M, Suarez D, et al. COVID-19 and Kawasaki disease: novel virus and novel case. Hosp Pediatr 2020;10:537-40.

2. Royal College of Paediatrics and Child Health. Guidance: Paediatric multisystem inflammatory syndrome temporally associated with COVID-19. Londres (Royaume-Uni); 2020. Accessible ici : www.rcpch.ac.uk/resources/guidance -paediatric-multisystem-inflammatory-syndrome-temporally-associated-covid -19 (consulté le 10 juin 2020).

3. Centers for Disease Control and Prevention. Multisystem inflammatory syndrome in children (MIS-C) associated with coronavirus disease 2019 (COVID-19). Atlanta; 14 mai 2020. Accessible ici : emergency.cdc.gov/han/2020/han00432.asp (consulté le 10 juin 2020).

4. Organisation mondiale de la Santé. Syndrome inflammatoire multisystémique chez les enfants et les adolescents atteints de COVID-19. Genève; 15 mai 2020. Accessible ici : apps.who.int/iris/bitstream/handle/10665/332190/WHO-2019-nCoV-Sci_Brief -Multisystem_Syndrome_Children-2020.1-fre.pdf?sequence=1\&isAllowed=y (consulté le 10 juin 2020).

5. Berard RA, Scuccimarri R, Haddad EM, et al. Le syndrome inflammatoire multisystémique de l'enfant ayant un lien temporel avec la COVID-19. Ottawa : Société canadienne de pédiatrie; 8 juillet 2020. Accessible ici : www.cps.ca/fr/ documents/position/SIME (consulté le 4 août 2020).
6. Programme canadien de surveillance pédiatrique. Alerte de santé publique : un lien temporel entre une maladie inflammatoire aiguë et la COVID-19 chez les enfants. Ottawa; 14 mai 2020. Accessible ici : www.pcsp.cps.ca/uploads/ private/PCSP_Alerte-sante-publique-maladie-inflammatoire-aigue-COVID_19.pdf (consulté le 10 juin 2020).

7. Whittaker E, Bamford A, Kenny J, et al. Clinical characteristics of 58 children with a pediatric inflammatory multisystem syndrome temporally associated with SARS-CoV-2. JAMA 2020;324:259-69.

8. Verdoni L, Mazza A, Gervasoni A, et al. An outbreak of severe Kawasaki-like disease at the Italian epicentre of the SARS-CoV-2 epidemic: an observational cohort study. Lancet 2020;395:1771-8.

9. Belhadjer Z, Méot M, Bajolle F, et al. Acute heart failure in multisystem inflammatory syndrome in children in the context of global SARS-CoV-2 pandemic. Circulation 2020;142:429-36.

10. Feldstein LR, Rose EB, Horwitz SM, et al. Multisystem inflammatory syndrome in U.S. children and adolescents. N Engl J Med 2020;383:334-46.

11. Varga Z, Flammer AJ, Steiger $P$, et al. Endothelial cell infection and endotheliitis in COVID-19. Lancet 2020;395:1417-8.

12. McCrindle BW, Rowley AH, Newburger JW, et al.; American Heart Association Rheumatic Fever, Endocarditis, and Kawasaki Disease Committee of the Council on Cardiovascular Disease in the Young; Council on Cardiovascular and Stroke Nursing; Council on Cardiovascular Surgery and Anesthesia; et Council on Epidemiology and Prevention. Diagnosis, treatment, and long-term management of Kawasaki disease: a scientific statement for health professionals from the American Heart Association. Circulation 2017;135:e927-9.

13. Wahezi DM, Lo MS, Rubinstein TB, et al. American College of Rheumatology guidance for the management of pediatric rheumatic disease during the COVID-19 pandemic: version 1. Arthritis Rheumatol 23 juillet 2020 [Cyberpublication avant impression]. doi : 10.1002/art.41455.

\section{Intérêts concurrents : Aucun déclaré.}

Cet article a été révisé par des pairs.

Les auteurs ont obtenu le consentement des parents.

Affiliation : Division de rhumatologie, Hôpital SickKids, Toronto, Ontario.

Collaborateurs : Rae Young a contribué à l'élaboration et à la conception de l'étude. Tous les auteurs ont participé à la collecte, à l'analyse et à l'interprétation des données et ont révisé de façon critique le contenu intellectuel important; ils ont donné leur approbation finale pour la version destinée à être publiée et assument l'entière responsabilité de tous les aspects du travail.

Financement : Rae Yeung est financée par la Chaire de recherche translationnelle en pédiatrie Hak-Ming et Deborah Chiu de l'Hôpital SickKids, Université de Toronto, Toronto, Ontario.

Correspondance : Rae Yeung, rae.yeung@sickkids.ca 